\title{
HOW FAR CAN ONE MOVE FROM A POTENTIAL PEAK WITH SMALL INITIAL SPEED?
}

\author{
ANGELO BARONE-NETTO AND GAETANO ZAMPIERI
}

(Communicated by Christopher Croke)

\begin{abstract}
We consider a natural Lagrangian system and show that from a point $q_{0}$ in $n$-space, where the potential energy $V$ has a (weak) maximum, one can go near the boundary of any compact ball where $V(q) \leq V\left(q_{0}\right)$ with (arbitrarily small) nonvanishing initial speeds. The result holds true for sets which are $C^{2}$-diffeomorphic to a compact ball. This property is found as a simple consequence of the Hopf-Rinow theorem and of a theorem of Gordon. As a corollary we deduce a well-known local result, namely, a 'converse' of the Lagrange-Dirichlet theorem, thus obtained via geometric arguments.
\end{abstract}

The present note deals with properties of the solutions to the Lagrange equations

$$
\frac{d}{d t} \frac{\partial L}{\partial \dot{q}}-\frac{\partial L}{\partial q}=0
$$

where the Lagrangian function is

$$
L \in C^{2}\left(\Omega \times \mathbb{R}^{n} ; \mathbb{R}\right), \quad \Omega=\Omega^{\circ} \subseteq \mathbb{R}^{n}, \quad L(q, \dot{q})=\frac{1}{2} \dot{q} \cdot g(q) \dot{q}-V(q)
$$

with symmetric matrix $g(q)>0$ at every $q \in \Omega$ and the centered dot is the Euclidean scalar product. One says that such a Lagrangian system is natural, and the function $V$ is called the potential energy. The function $\frac{1}{2} \dot{q} \cdot g(q) \dot{q}+V(q)$ is a first integral called the energy.

Assume that $q_{0} \in \Omega$ is a point of (weak) maximum, the ball $B\left[q_{0}, r_{0}\right]=$ $\left\{q:\left|q-q_{0}\right| \leq r_{0}<\infty\right\} \subset \Omega$, and

$$
V(q) \leq V\left(q_{0}\right) \quad \forall q \in B\left[q_{0}, r_{0}\right] .
$$

It is well known that (in an open set where $E-V(q)>0$ ) the geodesics of the Jacobi metric $(E-V(q)) g(q)$ give all the solutions to the Lagrange equations with energy equal to the constant $E$, up to reparametrization.

Received by the editors July 27, 1992 and, in revised form, September 16, 1992.

1991 Mathematics Subject Classification. Primary 34D20; Secondary 70G05.

Key words and phrases. Natural Lagrangian systems, Hopf-Rinow theorem.

The first author visited the University of Padova supported by the GNFM of the CNR, "Consiglio Nazionale delle Ricerche". Moreover, he received travel support from the FAPESP, "Fundação de Amparo à Pesquisa do Estado de São Paulo". The second author was supported by the CNR. 
Fix $E>V\left(q_{0}\right)$ and $r \in\left(0, r_{0}\right)$; moreover, consider a real-valued $C^{3}$ function $k>0$ defined on the open ball $B\left(q_{0}, r_{0}\right)=B\left[q_{0}, r_{0}\right]^{\circ}$ and such that

$$
k(q)=1 \quad \text { for } q \in B\left[q_{0}, r\right] \quad \text { and } \quad k(q) \rightarrow+\infty \quad \text { as }\left|q-q_{0}\right| \rightarrow r_{0} .
$$

So $k$ is a proper function; namely, the inverse image of any compact set is compact. By a theorem of Gordon (see the Corollary in Gordon [1]) we have completeness for the manifold $B\left(q_{0}, r_{0}\right)$ endowed with the Riemannian metric

$$
\exp \left(\frac{k^{\prime}(q) \cdot g(q)^{-1} k^{\prime}(q)}{E-V(q)}\right)(E-V(q)) g(q) .
$$

That is the geodesics are all defined on the whole of $\mathbb{R}$. In formula (5), $k^{\prime}(q)$ is the gradient of $k$ at $q$ and $g(q)^{-1}$ is the inverse matrix of $g(q)$. Now, let us invoke the Hopf-Rinow theorem to say that any two points of the aforementioned complete manifold are connected by a geodesic (see, e.g., Hermann [3]). The complete metric in formula (5) coincides with the Jacobi metric on $B\left[q_{0}, r\right]$; therefore, for each $\bar{q} \in B\left(q_{0}, r\right)$, there is a solution $q(\cdot)$ to the Lagrange equations such that

(i) $q(0)=\bar{q}$,

(ii) $E=\frac{1}{2} \dot{q}(0) \cdot g(\bar{q}) \dot{q}(0)+V(\bar{q})>V\left(q_{0}\right)$,

(iii) $q\left(t_{r}\right) \in \partial B\left[q_{0}, r\right]$ (the boundary of the ball) for some $t_{r}>0$, and

(iv) $q(t) \in B\left(q_{0}, r\right)$ for all $t<t_{r}$.

Since $r$ can be chosen arbitrarily near $r_{0}$, for every $\epsilon>0$ there is a solution $q(\cdot):[0, \hat{t}] \rightarrow B\left(q_{0}, r_{0}\right)$ with energy $E$ such that

$$
q(0)=\bar{q}, \quad \operatorname{dist}\left(q(\hat{t}) ; \partial B\left[q_{0}, r_{0}\right]\right)<\epsilon,
$$

where dist is the distance.

To get the previous result, we have exploited condition (3) (to have a Riemannian metric on the whole ball), and that condition does not depend on the kinetic energy function $\frac{1}{2} \dot{q} \cdot g(q) \dot{q}$. We can perform a $C^{2}$-diffeomorphism $Q(q)$ (defined on $\Omega$ ), and we can consider the new potential energy $V \circ Q^{-1}$. This yields the following statement.

Theorem. Let us consider a Lagrangian system as in (1) and (2), let $K \subset \Omega$ be a compact set $C^{2}$-diffeomorphic to a compact ball, and let $q_{0} \in K^{\circ}$. If $V \mid K$ has a global (weak) maximum at $q_{0}$, then for every choice of $E>V\left(q_{0}\right)$ and $\bar{q} \in K$ there exists a sequence of solutions $\left\{q_{n}(\cdot):\left[0, t_{n}\right] \rightarrow K\right\}$ with energy $E$ such that

$$
q(0)=\bar{q}, \quad \operatorname{dist}\left(q_{n}\left(t_{n}\right) ; \partial K\right) \rightarrow 0 \quad \text { as } n \rightarrow \infty .
$$

The abstract shows a direct consequence, easier to be stated than the theorem itself.

We conclude by finding a known result in Stability as a corollary of the (global) theorem above.

According to the celebrated Lagrange-Dirichlet theorem, if at a certain point $q_{0}$ the potential energy has a strict minimum, then the equilibrium solution $q(t)=q_{0}$ is stable. This sufficient condition for stability is not necessary, and some research is still devoted to finding sufficient conditions for instability (see Kozlov [4], Maffei, Mauro, and Negrini [5], and the references contained therein). Hagedorn [2] proved that instability occurs whenever $q_{0}$ is a strict 
maximum via a variational theorem of Caratheodory. In a second time, Taliaferro [6] generalized Hagedorn's result to a weak maximum (and to weaker regularity hypotheses). As a corollary of the theorem above, we have the instability of a weak maximum via purely geometric arguments.

\section{REFERENCES}

1. W. B. Gordon, An analytical criterion for the completeness of Riemannian manifolds, Proc. Amer. Math. Soc. 37 (1973), 221-225.

2. P. Hagedorn, Die umkehrung der stabilitätssätze von Lagrange-Dirichlet und Routh, Arch. Rational Mech. Anal. 42 (1971), 281-316.

3. R. Hermann, Differential geometry and the calculus of variations, Academic Press, New York, 1968.

4. V. V. Kozlov, Asymptotic motions and the inversion of the Lagrange-Dirichlet theorem, Prikl. Mat. Mekh. 50 (1986), 719-725.

5. C. Maffei, V. Moauro, and P. Negrini, On the inversion of the Lagrange-Dirichlet theorem in a case of nonhomogeneous potential, Differential Integral Equations 4 (1991), 767-782.

6. S. D. Taliaferro, An inversion of the Lagrange-Dirichlet stability theorem, Arch. Rational Mech. Anal. 73 (1980), 183-190.

Universidade de São Paulo, Instituto de Matematica e Estatistica, C. P. 20570, 01452 990 São Paulo, Brazil

E-mail address: barone@ime.usp.br

Universita di Padova, Dipartimento di Matematica Pura e Applicata, via Belzoni 7 , 35131 Padova, Italy

E-mail address: gaetano@pdmat1.unipd.it 